\title{
Autoidentificación de la cintura-útil en la ubicación del sitio de punción en anestesia neuroaxial de paciente obstétrica con obesidad clase III
}

\section{Self-identification of the waist - useful in the location of the puncture site in neuroaxial anesthesia of obstetric patient with class III obesity}

Dra. Martha Isabel Gaona-Ramírez*

Citar como: Gaona-Ramírez MI. Autoidentificación de la cintura-útil en la ubicación del sitio de punción en anestesia neuroaxial de paciente obstétrica con obesidad clase III. Rev Mex Anestesiol. 2021; 44 (4): 250-257. https://dx.doi. org/10.35366/100869

RESUMEN. La técnica anestésica neuroaxial en la paciente embarazada obesa clase III puede representar un reto para el anestesiólogo, debido a la dificultad para reconocer las referencias anatómicas. El objetivo del estudio fue determinar la dificultad y la calidad de la anestesia combinada espinal/epidural, en 14 pacientes embarazadas con índice de masa corporal $\geq 40 \mathrm{~kg} / \mathrm{m}^{2}$, programadas para cesárea. Para la localización del sitio de punción, se utilizó la autoidentificación de cintura, metodología no reportada previamente. Las pacientes se sentaron a horcajadas en la mesa quirúrgica, señalaron el lugar de su cintura con sus manos. Se trazaron dos rectas en la espalda, una recta horizontal en la cintura señalada por la paciente, y una recta vertical correspondiente a la línea media. La intersección de las dos rectas fue la coordenada para la punción neuroaxial. La media $\pm \mathrm{DE}$ del índice de masa corporal fue $47.5 \pm 3.7 \mathrm{~kg} / \mathrm{m}^{2}$. Se determinó una tasa de éxito del primer nivel del $92.8 \%$, un promedio de punciones de 1.5 y $57.1 \%$ de las pacientes recibió una punción. La anestesia espinal/epidural falló en dos casos, se realizó cambio de técnica anestésica a epidural, y complementación con infiltración local, con adecuada analgesia. No fue necesario convertir a anestesia general en ningún caso. La autoidentificación de la cintura puede ser una ayuda clínica para la identificación del sitio de la punción lumbar durante la instalación de la anestesia neuroaxial, en pacientes obstétricas con obesidad clase III programadas para cesárea.

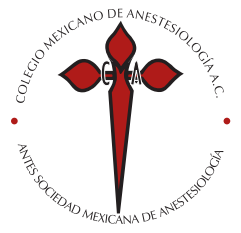

Palabras clave: Cintura, coordenada, punción, anestesia, neuroaxial, obesidad, cesárea.

Keywords:

Waist, coordinate, puncture, anesthesia, neuraxial, obesity, cesarean section.

* Maestra en Gestión de Calidad en Servicios de Salud, Instituto Nacional de Salud Pública. Médico Especialista en Anestesiología, UNAM, IMSS. Médico General, 1ra. Universidad Estatal de Medicina Ivan Petrovich Pávlov de San Petersburgo, Rusia.

Correspondencia:

Dra. Martha Isabel

Gaona-Ramírez

Ixtapan del Oro Núm. 67, Cumbria, 54740, Cuautitlán Izcalli, Estado de México, México. E-mail: lanegraeli@yahoo.com.mx

Recibido: 13-09-2019 Aceptado: 06-07-2020

ABSTRACT. The neuraxial anesthetic technique in the obese class III, pregnant patient, can represent a challenge for the anesthesiologist, due to the difficulty in recognizing the anatomical landmarks. The objective of the study was to determine the difficulty and quality of combined spinal/epidural anesthesia, in 14 pregnant patients with a body mass index $\geq 40 \mathrm{~kg} / \mathrm{m}^{2}$ scheduled for cesarean section. For the location of the puncture site, self-identification of the waist was used, a methodology that has not been previously reported. The patients straddled the surgical table, pointing to her waist with both hands. Two lines were drawn on the back. A horizontal line at the waist, indicated by the patient, and a vertical line corresponding to the midline. The intersection of the two lines was the coordinate for the neuraxial puncture. The mean $\pm S D$ of the body mass index was $47.5 \pm 3.7 \mathrm{~kg} / \mathrm{m}^{2}$. A first level success rate of $92.8 \%$ was determined, $57.1 \%$ of the patients received one puncture, and the average of punctures was 1.5. Spinal/epidural anesthesia failed in two cases, a change from anesthetic technique to epidural and supplementation with infiltration was performed, with adequate analgesia. It was not necessary to convert to general anesthesia in any case. Self-identification of the waist and coordinate marking can be a clinical aid for the identification of the lumbar puncture site, during the installation of neuraxial anesthesia, in obstetric patients with class III obesity, scheduled for cesarean section.

\section{INTRODUCCIÓN}

$\mathbf{L}$ a obesidad se define como una acumulación excesiva de $\_$grasa que puede ser perjudicial para la salud. El índice de masa corporal (IMC), definido como el peso de una persona en kilogramos dividido por el cuadrado de su altura en metros $\left(\mathrm{kg} / \mathrm{m}^{2}\right)$, es frecuentemente utilizado para clasificar la obesidad. Obesidad clase I (IMC entre 30 y $34.9 \mathrm{~kg} / \mathrm{m}^{2}$ ); obesidad clase II (IMC entre 35 y $39.9 \mathrm{~kg} / \mathrm{m}^{2}$ ); obesidad clase III (IMC $\geq 40 \mathrm{~kg} /$ $\left.\mathrm{m}^{2}\right)^{(1)}$. La prevalencia de obesidad en mujeres en edad fértil es preocupante por la asociación entre el IMC y las complicaciones maternas y fetales y el incremento del riesgo para la cesárea ${ }^{(2,3)}$. 
Amenos que exista alguna contraindicación, la técnica anestésica recomendada para la cesárea es la anestesia neuroaxial ${ }^{(4)}$. En la paciente embarazada con obesidad clase III, esta técnica puede ser un reto para el anestesiólogo. La dificultad para reconocer los reparos anatómicos que comúnmente se usan al determinar el sitio de punción en la región lumbar (línea media vertebral, apófisis espinosas, espacios intervertebrales, borde de la parrilla costal, crestas ilíacas, línea inter-crestas o de Tuffier) puede conducir a múltiples punciones o complicaciones anestésicas ${ }^{(5-10)}$.

La identificación de las apófisis espinosas como referencia anatómica de la línea media es un componente primordial durante la realización del bloqueo neuroaxial, puesto que representa el reparo anatómico que mejor predice la dificultad durante la aplicación del bloqueo anestésico ${ }^{(6,7,11)}$. Se ha reportado una tasa de éxito del primer nivel durante la anestesia neuroaxial (éxito en la localización del espacio espinal o epidural en el primer sitio de punción seleccionado) en el grupo de pacientes con procesos espinosos fácilmente palpables, de $89 \%$, contra $71 \%$ en el grupo donde no se pudo identificar positivamente las apófisis espinosas $(\mathrm{p}<0.02)^{(7)}$. Para determinar la línea media en la paciente obesa, se ha sugerido colocar a la paciente en posición sentada, observar la prominencia de la séptima vértebra cervical y la hendidura glútea ${ }^{(5,12)}$; preguntar a la paciente si siente la punción en la línea media $^{(13)}$ y durante la infiltración de la piel, identificar el espacio intervertebral tocando las apófisis espinosas superior e inferior con una aguja espinal 26 gauge, $8.5 \mathrm{~cm}^{(14)}$.

La determinación del nivel lumbar de la punción es el segundo aspecto que se debe tener en cuenta durante la técnica combinada espinal-epidural. Se ha recomendado utilizar el espacio entre las vértebras lumbares L3-L4 para evitar lesionar la médula espinal, que en el $19 \%$ de los pacientes termina debajo de $\mathrm{L1}^{(15)}$. En las embarazadas con obesidad clase III se ha sugerido que la visualización de la línea de Blass (línea imaginaria que pasa a través del pliegue que forman las almohadillas de tejido adiposo a nivel toracolumbar y corresponde al espacio entre las vértebras T11-T12) ayudaría a determinar el nivel del bloqueo cuando las referencias óseas se pierden debido al panículo adiposo ${ }^{(5)}$.

Desde hace varias décadas, se ha reconocido la circunferencia de la cintura como un marcador independiente de morbilidad y mortalidad $^{(1,16)}$. El protocolo descrito por las guías de la Organización Mundial de la Salud señala la cintura como el punto medio entre el borde inferior de la parrilla costal y el borde superior de la cresta ilíaca ${ }^{(17)}$; mientras que el protocolo de los institutos nacionales de salud la señala en el borde superior de la cresta ilíaca ${ }^{(18)}$. Se ha determinado que las mediciones de la cintura con estos dos protocolos concuerdan, son confiables y factibles para la realización por el público en general y el personal de salud ${ }^{(16)}$. La automedición de la cintura por el paciente tiene una correlación fuerte con la medición realizada por los técnicos ${ }^{(16,19)}$.

No existen reportes que describan la autoidentificación de la cintura para la localización del sitio de punción lumbar durante la anestesia neuroaxial en pacientes con obesidad clase III.
El objetivo de este estudio fue describir la metodología de ubicación del sitio de punción para anestesia neuroaxial para cesárea en pacientes embarazadas con obesidad clase III con el uso de la autoidentificación de la cintura, y determinar la dificultad de instalación de la anestesia espinal/epidural con técnica aguja a través de aguja, y la calidad de la anestesia neuroaxial.

\section{MATERIAL Y MÉTODOS}

Se realizó un estudio observacional, descriptivo, prospectivo, tipo serie de casos en un centro académico de tercer nivel de atención obstétrica. La muestra fue seleccionada por conveniencia de la población de pacientes atendidas en el turno nocturno, los días lunes, miércoles y viernes. Se incluyó a pacientes embarazadas, con integridad neurológica, obesidad clase III, programadas para cesárea, en quienes la técnica anestésica sugerida fue la anestesia combinada espinal/epidural con técnica aguja Whitacre a través de aguja Tuohy. Se realizó valoración preanestésica, evaluación de las referencias anatómicas para punción lumbar según Sprung (buenas, apófisis espinosas fácilmente palpables; deficientes, dificultad para palpar apófisis espinosas; ninguna, incapacidad para identificar las apófisis espinosas) ${ }^{(7)}$, asignación de riesgo anestésico-quirúrgico y de estado físico según la Sociedad Americana de Anestesiología. Se llevó a cabo consentimiento informado y manejo anestésico de acuerdo con la normatividad vigente ${ }^{(20-22)}$.

Se estableció una comunicación efectiva con la paciente, con el objetivo de facilitar su relajación y cooperación; así como, con el personal de quirófano para lograr la colaboración con el anestesiólogo en la movilización y posicionamiento de la paciente en la mesa quirúrgica ${ }^{(23)}$. Se colocó catéter nasal para administración de oxígeno y se mantuvo la monitorización no invasiva y la permeabilidad de las vías venosas durante la instalación del bloqueo nervioso.

La metodología de ubicación del sitio de punción lumbar inició con la colocación de la paciente sentada a horcajadas, centrada en la mesa quirúrgica, con la espalda erguida y la mirada al frente (Figura 1). No se realizó palpación de la espalda de la paciente para evitar incomodidad y dolor, tratando de encontrar referencias óseas que ya habían sido evaluadas y no fueron encontradas. Se pidió a la paciente que señalara su cintura, colocando sus manos a los lados del cuerpo con los pulgares hacia atrás (Figura 2). A continuación, la autora del presente reporte trazó con un marcador una recta horizontal que unió la mitad de los dos pulgares (Figura 3). Después se identificó la línea media, según las recomendaciones de la literatura. A las pacientes, en quienes el surco interglúteo estuvo oculto por el panículo adiposo, se les pidió inclinarse hacia delante para visualizar el origen del mismo. Se trazó una recta vertical (perpendicular al plano de la mesa quirúrgica y a la línea horizontal previamente trazada). El punto de intersección de las dos rectas fue la coordenada determinada 
como sitio de punción para la anestesia neuroaxial en todas las pacientes (Figura 4).

Se le pidió a la paciente flexionar su cabeza sobe el pecho y se procedió a la instalación de la anestesia combinada espinalepidural por los médicos anestesiólogos del servicio. Se utilizó la técnica de pérdida de la resistencia para localización del espacio epidural con aguja Tuohy, y la técnica aguja a través de aguja para la localización del espacio subaracnoideo con aguja Whitacre, según están descritas en la literatura. La permeabilidad del catéter epidural se corroboró en todos los casos, y los medicamentos utilizados fueron seleccionados por cada médico tratante, según las condiciones de la paciente ${ }^{(15)}$.

Se determinó la dificultad de la instalación de la anestesia neuroaxial a través del éxito del primer nivel y del número de punciones. El éxito del primer nivel se definió como la localización del espacio espinal/epidural, en el sitio de punción marcado con la coordenada determinada con la metodología de autoidentificación de la cintura. El número de punciones se definió por el número de veces que la aguja de Tuohy atravesó la piel en el intento de localizar el espacio epidural, independientemente de si se realizó en el sitio determinado por coordenada o si se tuvo que cambiar de sitio.

Se registró el éxito del bloqueo neuroaxial. Se consideró que la anestesia combinada espinal-epidural falló cuando fue necesario complementar con infiltración de anestésico local, realizar un segundo bloqueo neuroaxial, o cambiar la técnica anestésica (uso exclusivo de anestesia espinal o de anestesia epidural, administración de anestesia general). Se registraron los eventos relacionados con la instalación de la anestesia neuroaxial, que se consideró de importancia para el manejo anestésico de las pacientes. Se presentan los resultados en media \pm DE y frecuencia proporcional.

\section{RESULTADOS}

En esta serie de casos se incluyeron 14 pacientes obstétricas con obesidad clase III, programadas para cesárea bajo anestesia combinada espinal-epidural. Todas las pacientes fueron atendidas en el turno nocturno por médicos anestesiólogos adscritos y en formación, con experiencia en el manejo anestésico de pacientes obstétricas. Las características demográficas de los casos incluidos se muestran en la Tabla 1. La media \pm DE del IMC fue $47.5 \pm 3.7$. En el caso 13, IMC $42 \mathrm{~kg} / \mathrm{m}^{2}$, se detectó escoliosis de la columna vertebral, evidenciado por un surco interglúteo no perpendicular a la mesa quirúrgica. La monitorización y posición de la paciente se ilustra en la Figura 1. Todas las pacientes entendieron las instrucciones expresadas por la autora del reporte, para señalar su cintura; la autoidentificación de la cintura se llevó a cabo sin que se presentaran eventualidades (Figura 2). Para determinar el nivel de la punción lumbar, se dibujó la recta horizontal en la espalda de las pacientes, como se ilustra en la Figura 3. No se utilizó la línea de Blass por la distribución irregular del tejido adiposo en la espalda. En algunos casos, para visualizar los pulgares de la paciente, fue necesario levantar los sacos adiposos laterales, lo que se logró exitosamente. En la Figura 4 se puede ver la recta vertical y el lugar de intersección de las dos rectas, que corresponde a la coordenada del sitio de punción para la instalación de la anestesia neuroaxial.

Para la instalación de la anestesia neuroaxial en todas las pacientes se utilizó aguja de Tuohy de longitud estándar. En algunos casos fue necesario realizar la instalación de la anestesia a cuatro manos: dos sosteniendo las alas de la aguja de Tuohy y presionando el panículo adiposo, y las otras dos colocando la aguja de Whitacre, el medicamento en espacio subaracnoideo y el catéter epidural.

El éxito del primer nivel fue del 92.8\%. La media \pm DE del número de punciones fue $1.5 \pm 0.65$; el $57.1 \%$ de los casos recibió una punción; el 37.1\%, dos; y el 7.1\%, tres. La anestesia regional se consideró satisfactoria en todas las pacientes, con una difusión del bloqueo a T4, analgesia adecuada y bloqueo motor presente. Sin embargo, la técnica espinal/epidural falló en dos pacientes. En el caso 4, IMC $47.3 \mathrm{~kg} / \mathrm{m}^{2}$, durante la instalación de la anestesia neuroaxial no se obtuvo líquido cefalorraquídeo y se cambió la técnica a bloqueo epidural.

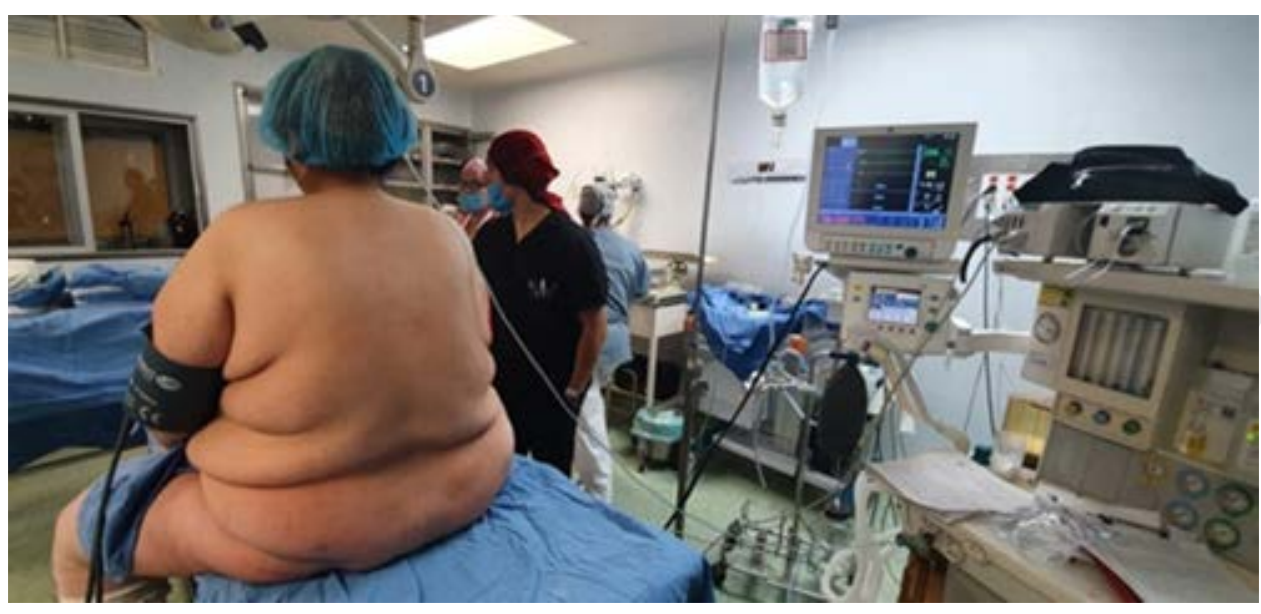

Figura 1:

Monitorización y posición de paciente. Caso 14, IMC $47.2 \mathrm{~kg} / \mathrm{m}^{2}$. (Publicada con permiso de la paciente). 


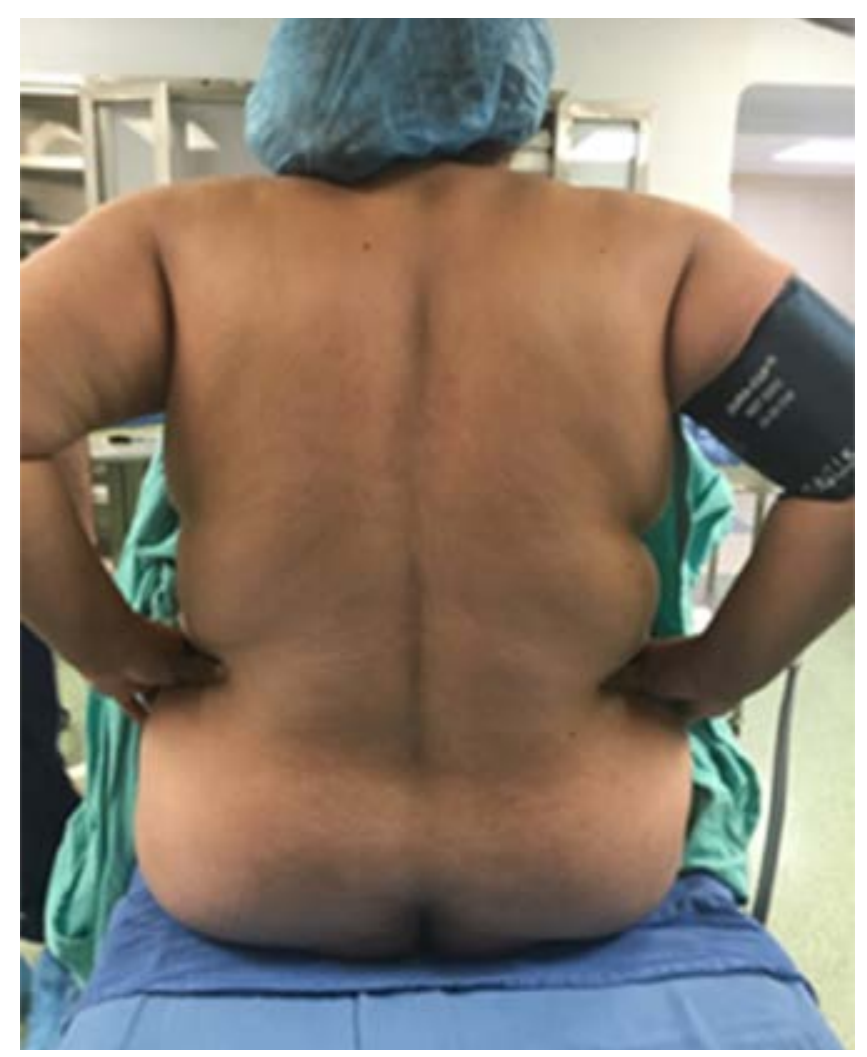

Figura 2: Autoidentificación de la cintura. Caso 6, IMC $47.5 \mathrm{~kg} / \mathrm{m}^{2}$. (Publicada con permiso de la paciente).

En el caso 8, IMC $54.4 \mathrm{~kg} / \mathrm{m}^{2}$, no fue posible administrar dosis de anestésico local por el catéter epidural al término de la cirugía (después del cierre de aponeurosis), por lo que se realizó infiltración del tejido celular subcutáneo. No se utilizó sedación ni anestesia general en ninguna paciente (Tabla 2).

\section{DISCUSIÓN}

La autoidentificación de la cintura como ayuda clínica para la localización del sitio de la punción lumbar durante la instalación de la anestesia neuroaxial ha demostrado ser útil en este grupo de pacientes obstétricas con obesidad clase III. Se reporta una tasa de éxito del primer nivel de 92.8\%, un promedio de punciones de 1.5, y más de la mitad de las pacientes recibió una punción.

Las características clínicas de las pacientes en esta serie de casos confirman que la obesidad se asocia con comorbilidades y complicaciones materno fetales ${ }^{(24)}$; y que las referencias anatómicas para la realización de la anestesia neuroaxial no son evidentes. Debido a que las pacientes embarazadas con obesidad clase III tienen cerca del triple de riesgo de requerir cesárea, comparadas con las pacientes con IMC normal ${ }^{(25,26)}$, los anestesiólogos requerirán herramientas útiles para la atención.
La metodología descrita incluye dos elementos de innovación con respecto a las descripciones previas para la instalación de la anestesia neuroaxial en paciente obstétrica obesa: la posición de la paciente, y la identificación del sitio lumbar de la punción con la autoidentificación de la cintura.

Aunque Nazar sugirió que la mejor posición de la paciente obesa para la anestesia regional en cesárea es la que domina el operador $^{(5)}$, se prefirió seguir la recomendación de mantener a la paciente sentada durante la instalación del bloqueo, porque la profundidad del espacio epidural es menor, y las almohadillas de grasa no obstruyen la visualización de la línea media, como lo hacen en la posición decúbito lateral ${ }^{(12)}$.

En este estudio se optó por la posición sentada a horcajadas en la mesa quirúrgica, que no ha sido reportado previamente. La posición sentada tradicional, en la cual las rodillas se flexionan aproximadamente $90^{\circ}$, los pies se apoyan en un soporte fijo y las caderas están en aducción ${ }^{(27)}$, requiere de un esfuerzo considerable debido a que el banco de apoyo existente en nuestro medio ofrece una superficie insuficiente para el apoyo de los pies, lo que le genera incomodidad para mantener las piernas juntas y dificultad para mantenerse quieta. La posición sentada con las piernas paralelas y estiradas sobre la mesa es recomendada en algunos $\operatorname{casos}^{(27,28)}$, pero no es la óptima en las pacientes con

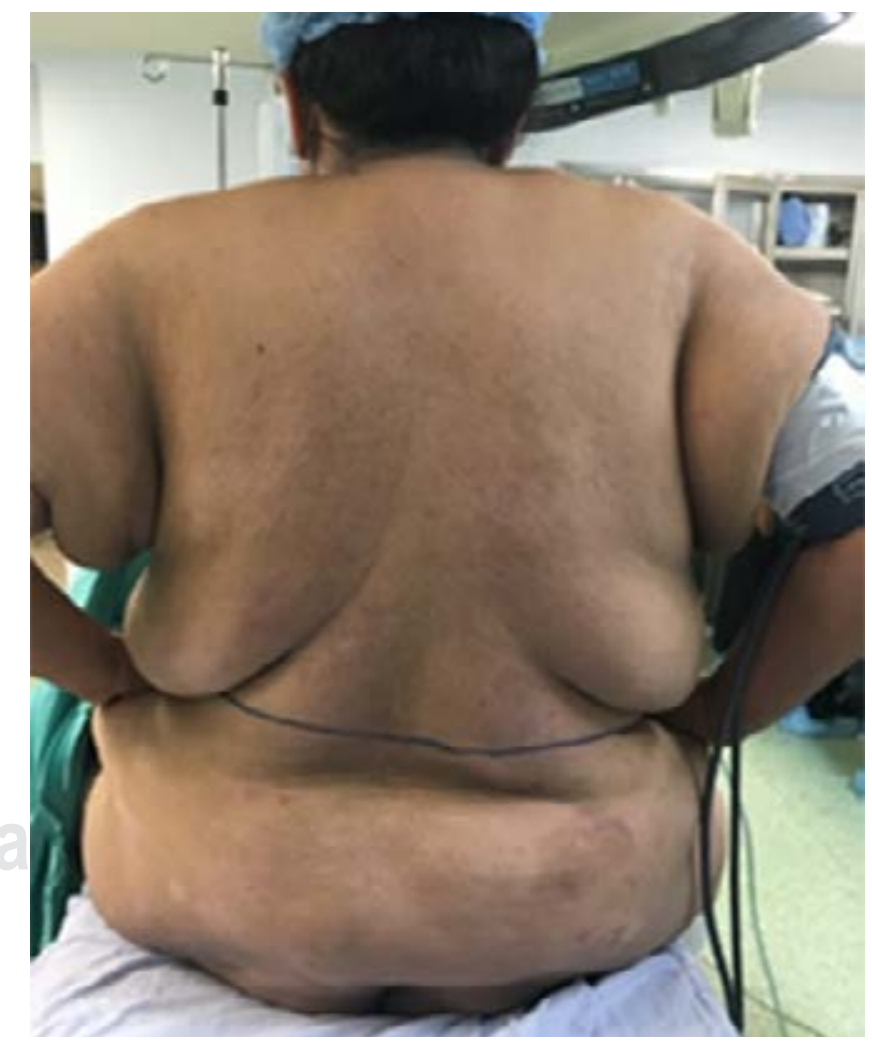

Figura 3: Identificación del nivel lumbar de la punción. Coordenada horizontal, caso 8, IMC $54.4 \mathrm{~kg} / \mathrm{m}^{2}$. (Publicada con permiso de la paciente). 


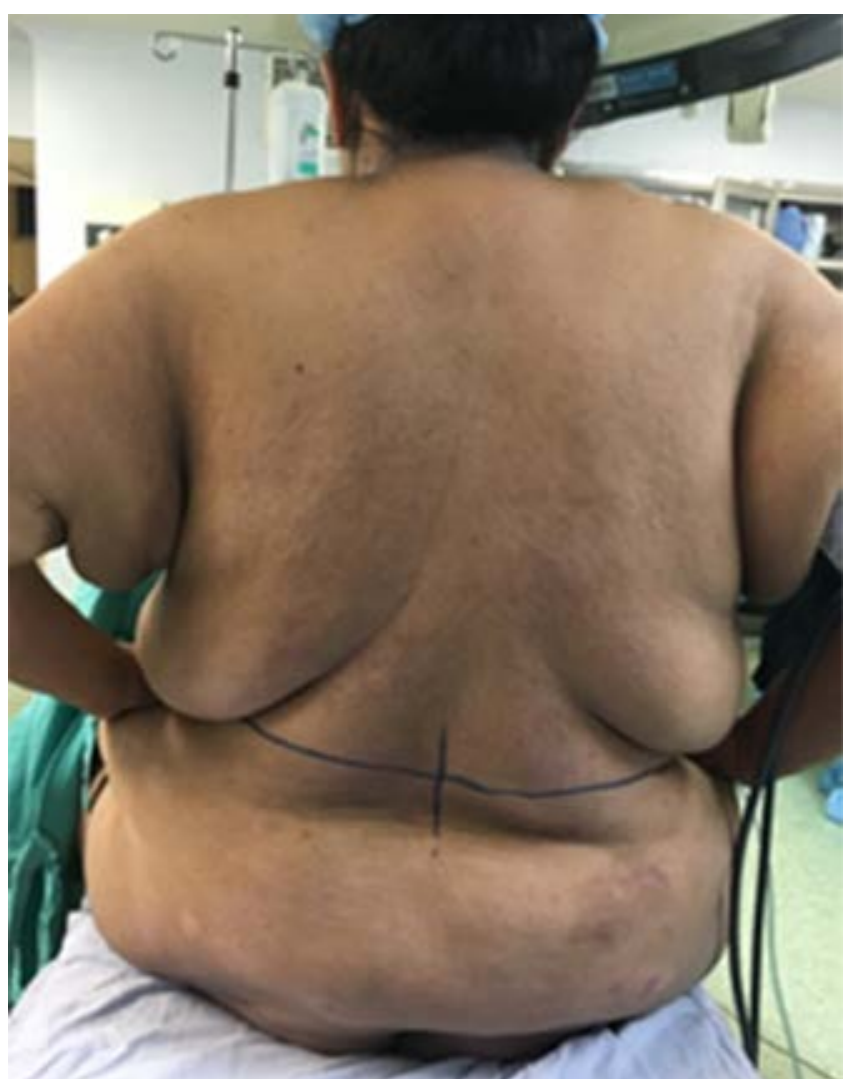

Figura 4: Identificación de línea media. Trazado de coordenada vertical, caso 8, IMC $54.4 \mathrm{~kg} / \mathrm{m}^{2}$. (Publicada con permiso de la paciente).

obesidad clase III, debido a que la mujer trata de compensar el peso del abdomen grávido extendiendo su cuerpo hacia atrás, y conduce a cansancio y movimientos durante la realización de la técnica regional. La posición sentada a horcajadas, que se propone en este reporte, es cómoda y disminuye los movimientos de la persona, permite que el abdomen grávido se apoye en la mesa y favorece la flexión de la paciente al momento de realizar la anestesia neuroaxial. Esta posición permite mantener el monitoreo no invasivo y las vías venosas accesibles y permeables, ya que los brazos descansan sobre los muslos de la paciente. Por último, la posición sentada a horcajadas en la mesa es muy conveniente para la movilización de la paciente una vez instalada la anestesia, permitiendo recolocarla con mayor facilidad en la posición supina sobre la mesa quirúrgica.

La autoidentificación de la cintura es el segundo elemento de innovación. Se fundamenta en la capacidad del individuo de señalar las partes de su cuerpo. La acción de señalar las partes del propio cuerpo alcanza la madurez del adulto entre los cuatro y cinco años de edad, e implica representar al cuerpo como objetivo de la acción y como efector de la misma ${ }^{(29)}$. La representación mental del esquema corporal se caracteriza por la plasticidad, por no estar representado en una sola región del cerebro y por integrar la información de diferentes modalidades sensoriales (táctil, propioceptiva, visual) para alcanzar una representación abstracta del cuerpo. También se categoriza por una codificación espacial, en el sentido de que representa la posición y la configuración del cuerpo como un objeto tridimensional en el espacio ${ }^{(29,30)}$. El esquema corporal en una persona neurológicamente íntegra permite que identifique las partes de su propio cuerpo.

Cada una de las mujeres identificó su cintura sin dificultad. De acuerdo con la definición de cintura de la Organización Mundial de la Salud y de los institutos nacionales ${ }^{(16,17)}$, el eje o recta horizontal trazada entre los dos pulgares (la cintura) se encuentra en la región lumbar. Reportar con precisión el nivel lumbar de la punción sería especulativo; sin embargo, esto no es una sorpresa. La instalación de la anestesia neuroaxial se caracteriza por ser una técnica a ciegas ${ }^{(15)}$. Se ha reportado que aún en pacientes en quienes fue posible palpar las referencias óseas, en especial las crestas ilíacas y la línea de Tuffier, la precisión del nivel espinal fue del $36.4 \%{ }^{(31)}$, y se conoce que existe un sesgo cefálico ${ }^{(31,32)}$.

El método que podría informar con mayor precisión el nivel donde se realiza la punción es el ultrasonido. Este es un método objetivo, operador dependiente, que se utiliza pre-punción o en tiempo real ${ }^{(33,34)}$, pero no está disponible en muchos centros.

La dificultad de la instalación de la técnica neuroaxial se ha descrito a través del éxito del primer nivel, el número de punciones, número de espacios intervertebrales «intentados», número de redirecciones, número de encuentros con hueso y tiempo de colocación del bloqueo neuroaxial ${ }^{(6,10,11)}$. En este estudio sólo se midieron dos parámetros: el éxito del primer nivel y el número de punciones. Debido a que la técnica neuroaxial es un procedimiento intuitivo basado en la sensibilidad táctil del anestesiólogo, la redirección de la aguja y cuantificación de encuentros óseos pueden ser subjetivas y difíciles de medir.

Con la autoidentificación de la cintura y el marcaje de coordenada, la dificultad para instalar la anestesia neuroaxial fue baja. Se cree que la mayor dificultad para instalar el bloqueo, en el caso 13 (IMC $42 \mathrm{~kg} / \mathrm{m}^{2}$ ), estuvo relacionada con la anormalidad estructural de la columna ${ }^{(7)}$. Se sugiere contar con agujas largas para uso en paciente obstétrica con obesidad clase III.

La obesidad es un problema de salud pública en México, con una prevalencia del $40.2 \%$ entre mujeres de 20 años y más ${ }^{(35)}$. En consecuencia, los retos que un anestesiólogo enfrentará al atender una paciente embarazada con obesidad clase III, programada para cesárea, no deberán ser subestimados. Tendrá que prever los riesgos, tomar decisiones sobre la marcha y resolver las complicaciones relacionadas con la técnica anestésica seleccionada ${ }^{(36,37)}$. En caso de presentarse fallo durante la instalación de la técnica neuroaxial recomendada, el anestesiólogo deberá estar preparado, si las condiciones obstétricas de la paciente lo permiten, para cambiar a otra técnica regional y/o rebloquear ${ }^{(15,36,38)}$. Entre las técnicas neuroaxiales 
alternativas, está la anestesia espinal con dosis única, espinal continua, epidural, espinal-epidural en diferentes niveles, y la técnica de doble catéter (epidural torácico + lumbar espinal) (24). La anestesia espinal con dosis única tiene el inconveniente que no se puede prolongar en el tiempo la duración del bloqueo neuroaxial, situación que puede requerir que se convierta a anestesia general. La anestesia espinal continua requiere una punción en espacio subaracnoideo con aguja de Tuohy y la introducción de un catéter para titular la dosis. Sin embargo, la punción de la duramadre con aguja gruesa puede provocar cefalea postpunción $^{(12,38)}$. La técnica de doble catéter se ha reportado de utilidad en tres pacientes con IMC $>73 \mathrm{~kg} / \mathrm{m}^{2(24)}$.

En la presente serie de casos, la anestesia combinada espinal/epidural falló en dos pacientes. En el caso 4, debido a

\begin{tabular}{|c|c|c|c|c|c|c|c|c|c|c|c|}
\hline Caso & $\begin{array}{l}\text { Edad } \\
\text { (años) }\end{array}$ & SDG & G & Diagnóstico & Antecedentes & Peso (kg) & Talla (m) & IMC $\left(\mathrm{kg} / \mathrm{m}^{2}\right)$ & $\begin{array}{l}\text { Referencias } \\
\text { anatómicas }\end{array}$ & ASA & Programación \\
\hline 1 & 36 & 38.5 & 3 & $\begin{array}{l}\text { Preeclampsia } \\
\text { sobreagregada }\end{array}$ & $\begin{array}{c}\text { HAS } \\
\text { Colecistectomía } \\
\text { Cesárea (2) }\end{array}$ & 125 & 1.70 & 43.3 & Deficiente & 4 & Urgencia \\
\hline 2 & 34 & 41.1 & 2 & Baja reserva fetal & $\begin{array}{c}\text { HAS } \\
\text { Cesárea (1) }\end{array}$ & 123 & 1.60 & 48.0 & Ninguna & 3 & Urgencia \\
\hline 3 & 38 & 39.0 & 3 & Oligohidramnios & $\begin{array}{c}\text { HAS } \\
\text { Parto (1) } \\
\text { Aborto (1) }\end{array}$ & 110 & 1.50 & 48.9 & Ninguna & 4 & Urgencia \\
\hline 4 & 37 & 37.5 & 4 & $\begin{array}{l}\text { Preeclampsia con } \\
\text { datos de severidad }\end{array}$ & $\begin{array}{l}\text { Cesárea (1) } \\
\text { Aborto (2) }\end{array}$ & 115 & 1.56 & 47.3 & Ninguna & 4 & Urgencia \\
\hline 5 & 24 & 38.5 & 2 & Cesárea iterativa & $\begin{array}{l}\text { Diabetes mellitus } \\
\text { gestacional } \\
\text { Cesárea (1) }\end{array}$ & 131 & 1.65 & 48.1 & Ninguna & 3 & Electiva \\
\hline 6 & 26 & 35.5 & 2 & $\begin{array}{l}\text { Ruptura de } \\
\text { membranas }\end{array}$ & $\begin{array}{l}\text { Hipotiroidismo } \\
\text { Parto (1) }\end{array}$ & 114 & 1.55 & 47.5 & Deficiente & 3 & Urgencia \\
\hline 7 & 30 & 38.1 & 2 & Cesárea iterativa & Cesárea (1) & 112 & 1.55 & 46.6 & Deficiente & 2 & Electiva \\
\hline 8 & 32 & 38.1 & 2 & $\begin{array}{l}\text { Falta de progresión } \\
\text { de trabajo de parto }\end{array}$ & $\begin{array}{c}\text { HAS } \\
\text { Diabetes mellitus } \\
\text { gestacional } \\
\text { Cesárea (1) }\end{array}$ & 141 & 1.61 & 54.4 & Ninguna & 3 & Urgencia \\
\hline 9 & 37 & 37.0 & 2 & $\begin{array}{l}\text { Preeclampsia con } \\
\text { datos de severidad }\end{array}$ & $\begin{array}{l}\text { Diabetes mellitus } \\
\text { gestacional } \\
\text { Cesárea (1) }\end{array}$ & 125 & 1.60 & 48.8 & Ninguna & 4 & Urgencia \\
\hline 10 & 32 & 38.0 & 2 & $\begin{array}{l}\text { Estado fetal no } \\
\text { confiable }\end{array}$ & $\begin{array}{c}\text { Hipotiroidismo } \\
\text { Parto (1) } \\
\text { Óbito }\end{array}$ & 135 & 1.57 & 54.8 & Ninguna & 3 & Urgencia \\
\hline 11 & 26 & 38.0 & 4 & Cesárea iterativa & $\begin{array}{c}\text { HAS } \\
\text { Hipotiroidismo } \\
\text { Cesárea (2) } \\
\text { Aborto (1) }\end{array}$ & 104 & 1.52 & 45.0 & Deficiente & 3 & Electiva \\
\hline 12 & 37 & 35.0 & 4 & Baja reserva fetal & $\begin{array}{c}\text { HAS } \\
\text { Hipotiroidismo } \\
\text { Diabetes mellitus } \\
\text { gestacional } \\
\text { Parto (1) } \\
\text { Aborto (2) } \\
\text { Estómago lleno }\end{array}$ & 109 & 1.58 & 43.7 & Deficiente & 4 & Urgencia \\
\hline 13 & 37 & 35.0 & 1 & $\begin{array}{l}\text { Inducción fallida de } \\
\text { trabajo de parto }\end{array}$ & $\begin{array}{l}\text { Hipotiroidismo } \\
\text { descontrolado }\end{array}$ & 99.5 & 1.54 & 42.0 & Ninguna* & 3 & Urgencia \\
\hline 14 & 37 & 35.0 & 2 & $\begin{array}{l}\text { Inducción fallida de } \\
\text { trabajo de parto }\end{array}$ & $\begin{array}{l}\text { Hipotiroidismo } \\
\text { descontrolado } \\
\text { Cesárea (1) } \\
\text { Cirugía bariátrica }\end{array}$ & 112 & 1.54 & 47.2 & Ninguna & 3 & Urgencia \\
\hline
\end{tabular}

* Explicación en el texto.

SDG = semanas de gestación; $\mathrm{G}=$ número de embarazos; $\mathrm{HAS}=$ hipertensión arterial sistémica; IMC = índice de masa corporal; ASA = estado físico de la Sociedad Americana de Anestesiólogos.

Referencias anatómicas: Sprung J, et al. ${ }^{7}$ 
Tabla 2: Resultados.

\begin{tabular}{|c|c|c|c|c|c|c|c|c|}
\hline \multirow[b]{2}{*}{ Caso } & \multirow[b]{2}{*}{ IMC $\left(\mathrm{kg} / \mathrm{m}^{2}\right)$} & \multicolumn{2}{|c|}{$\begin{array}{l}\text { Dificultad para instalación de la } \\
\text { anestesia neuroaxial }\end{array}$} & \multicolumn{3}{|c|}{$\begin{array}{c}\text { Éxito de la anestesia combinada } \\
\text { espinal/epidural }\end{array}$} & \multicolumn{2}{|c|}{$\begin{array}{c}\text { Complicaciones anestésicas relaciona- } \\
\text { das con el procedimiento }\end{array}$} \\
\hline & & $\begin{array}{l}\text { Éxito del } \\
\text { primer nivel* }\end{array}$ & $\begin{array}{l}\text { Número de } \\
\text { punciones }{ }^{\star *}\end{array}$ & $\begin{array}{l}\text { Anestesia espinal/ } \\
\text { epidural satisfactoria }\end{array}$ & $\begin{array}{l}\text { Cambio de técnica } \\
\text { anestésica }\end{array}$ & $\begin{array}{l}\text { Anestesia } \\
\text { satisfactoria }\end{array}$ & $\begin{array}{c}\text { Punción advertida } \\
\text { de duramadre con } \\
\text { aguja Tuohy }\end{array}$ & $\begin{array}{c}\text { Cefalea } \\
\text { postpunción } \\
\text { de duramadre }\end{array}$ \\
\hline 1 & 43.3 & Sí & 1 & Sí & No & Sí & No & No \\
\hline 2 & 48.0 & Sí & 1 & Sí & No & Sí & No & No \\
\hline 3 & 48.9 & Sí & 1 & Sí & No & Sí & No & No \\
\hline 4 & 47.3 & Sí & 1 & No & Epidural & Sí & No & No \\
\hline 5 & 48.1 & Sí & 1 & Sí & No & Sí & No & No \\
\hline 6 & 47.5 & Sí & 2 & Sí & No & Sí & No & No \\
\hline 7 & 46.6 & Sí & 2 & Sí & No & Sí & No & No \\
\hline 8 & 54.4 & Sí & 2 & No & Infiltración Subcutánea & Sí & No & No \\
\hline 9 & 48.8 & Sí & 2 & Sí & No & Sí & Sí & No \\
\hline 10 & 54.8 & Sí & 1 & Sí & No & Sí & No & No \\
\hline 11 & 45.0 & Sí & 1 & Sí & No & Sí & No & No \\
\hline 12 & 43.7 & Sí & 1 & Sí & No & Sí & No & No \\
\hline 13 & 42.0 & No & 3 & Sí & No & Sí & No & No \\
\hline 14 & 47.2 & Sí & 2 & Sí & No & Sí & No & No \\
\hline
\end{tabular}

* Localización de espacio espinal/peridural en el sitio de punción marcado con la coordenada obtenida con la autoidentificación de la cintura.

${ }^{* *}$ Cada vez que la aguja de Tuohy atraviesa la piel.

IMC = indice de masa corporal.

que no se obtuvo líquido cefalorraquídeo a través de la aguja Whitacre, se tuvo que cambiar la técnica a anestesia epidural. Se ha reportado que la falla para obtener líquido cefalorraquídeo a través de la aguja espinal se debe al uso de una aguja muy corta, de diámetro pequeño, o con falta de rigidez para atravesar la dura. Se pudo haber presentado desviación de la línea media durante la punción y aunque se identificó el espacio epidural, la aguja espinal pudo pasar por el lado de la dura ${ }^{(15)}$. En el caso 8, la dosis subaracnoidea inicial funcionó en forma adecuada, el catéter epidural se instaló y se comprobó su permeabilidad sin problema, proporcionando una anestesia suficiente por la mayor parte del procedimiento quirúrgico. Sin embargo, no fue posible administrar dosis epidural complementaria para mantener la anestesia quirúrgica. Se consideró que los movimientos de reposicionamiento de la paciente sobre la mesa quirúrgica después de instalada la anestesia neuroaxial en forma exitosa, pudieron provocar que el catéter epidural se saliera. Debido a que, cuando la paciente se flexiona para la instalación del bloqueo el espacio epidural se acerca a la piel ${ }^{(12)}$, se ha recomendado dejar en espacio epidural una longitud mayor a $4 \mathrm{~cm}$ de catéter para evitar esta complicación ${ }^{(38)}$.

En caso de que la calidad del bloqueo neuroaxial sea insuficiente o que sea imposible la instalación de la técnica anestésica regional, el anestesiólogo tendrá que considerar el cambio a anestesia general, con las implicaciones que tiene esta decisión. La administración de anestesia general se asocia con mayor morbilidad para los casos por las complicaciones derivadas por el manejo de la vía aérea ${ }^{(8,12,24,38)}$ y mayor riesgo para el anestesiólogo a la exposición a patógenos, entre ellos SARS-CoV-2, por la realización de procedimientos generadores de aerosoles ${ }^{(39)}$.

En este grupo de estudio, la calidad de la anestesia regional fue adecuada en todas las pacientes y no fue necesario convertir a anestesia general.

Los resultados obtenidos en esta investigación han sido positivos, pero deberán analizarse con cautela. Si bien, este formato de análisis permitió reportar dos elementos de innovación, la falta de aleatorización y control podría implicar que los resultados no se repliquen. Se requerirán en un futuro estudios controlados que validen la técnica de autoidentificación de cintura para identificación del sitio de punción lumbar en anestesia neuroaxial.

\section{CONCLUSIÓN}

La autoidentificación de la cintura en esta serie de casos ha facilitado la ubicación del sitio de la punción para la anestesia neuroaxial en embarazada con obesidad clase III programada para cesárea. Esta herramienta clínica podría agregarse al arsenal de conocimiento y habilidad de un anestesiólogo, en especial de quienes están en formación, y contribuir al manejo anestésico y confort de la paciente obstétrica en contextos donde no hay personal experto a quién recurrir, y donde el equipo de ultrasonido no esté disponible. 


\section{REFERENCIAS}

1. NICE National Institute for Health and Care Excellence. Obesity: identification, assessment and management. Clinical guideline CG189 [Internet]. 2014. Available from: https://www.nice.org.uk/guidance/cg189

2. ACOG Practice Bulletin No 156: obesity in pregnancy. Obstet Gynecol. 2015;126:e112-e126.

3. CMACE Centre for Maternal and Child Enquirires, RCOG Royal College of Obstetricians and Gynaecologists. CMACE/RCOG Joint Guideline. Management of women with obesity in pregnancy [Internet]. 2010. Available from: https://www.oaa-anaes.ac.uk/assets/_managed/editor/File/ Reports/2010_CMACE-RCOG_guideline_obesity_in_pregnancy.pdf

4. Practice Guidelines for Obstetric Anesthesia: An Updated Report by the American Society of Anesthesiologists Task Force on Obstetric Anesthesia and the Society for Obstetric Anesthesia and Perinatology. Anesthesiology. 2016;124:270-300.

5. Nazar C, Bastidas J, Zamora M, Lacassie H. Obesidad y embarazo: implicaciones anestésicas. Rev Chil Obs Ginecol. 2014;79:537-545.

6. Ellinas E, Eastwood D, Patel S, Maitra-D’Cuze A, Ebert T. The effect of obesity on neuraxial technique difficulty in pregnant patients: a prospective, observational study. Anesth Analg. 2009;109:1225-1231.

7. Sprung J, Bourke D, Grass J, Hammel J, Mascha E, Thomas P, et al. Predicting the difficult neuraxial block: a prospective study. Anesth Analg. 1999;89:384-389.

8. Bamgbade OA, Khalaf WM, Ajai O, Sharma R, Chidambaram V, Madhavan G. Obstetric anaesthesia outcome in obese and non-obese parturients undergoing caesarean delivery: an observational study. Int J Obstet Anesth. 2009;18:221-225.

9. Rodrigues FR, Brandao MJ. Regional anesthesia for cesarean section in obese pregnant women: a retrospective study. Rev Bras Anestesiol. 2011;61:13-20.

10. Kula AO, Riess ML, Ellinas EH. Increasing body mass index predicts increasing difficulty, failure rate, and time to discovery of failure of epidural anesthesia in laboring patients. J Clin Anesth. 2017;37:154-158.

11. Palencia M, Guasch E, Navas D, Gisanz F. Difficulty of epidural puncture for obstetric analgesia: risk factors. Rev Esp Anestesiol Reanim. 2006;53:139-144.

12. Lamon A, Habib A. Managing anesthesia for cesarean section in obese patients: current perspectives. Local Reg Anesth [Internet]. 2016 [cited 2020 Aug 6];9:45-57. Available from: https://www.dovepress.com/ managing-anesthesia-nbspfor-cesarean-section-in-obese-patientscurrent-peer-reviewed-article-LRA

13. Wills J, Bowie R, Bogod D. A pilot study of patient-led identification of the midline of the lumbar spine. Anaesthesia. 2002;57:390-394.

14. Maitra A, Palmer S, Bachhuber S, Abram S. Continuous epidural analgesia for cesarean section in a patient with morbid obesity. Anesth Analg. 1979;58:348-349.

15. Ranasinghe J, Davidson E, Birnbach D. Combined spinal-epidural anesthesia [Internet]. NYSORA - The New York School of Regional Anesthesia. [cited 2020 Aug 8]. Available from: https://www.nysora. com/regional-anesthesia-for-specific-surgical-procedures/abdomen/ combined-spinal-epidural-anesthesia/

16. Ross R, Neeland I, Yamashita S, Shai I, Seidell J, Magni P, et al. Waist circumference as a vital sign in clinical practice: a consensus statement from the IAS and ICCR working group on visceral obesity. Nat Rev Endocrinol. 2020;16:177-189.

17. WHO World Health Organization. Physical status: the use and interpretation of anthropometry: report of a WHO Expert Committee. Geneva, Switzerland: WHO; 1995.

18. NHLBI Obesity Education Initiative. The practical guide to the identification, evaluation and treatment of overweight and obesity in adults [Internet]. 2000. Available from: https://www.nature.com/articles/s41574-019-0310-7

19. Spencer E, Roddam A, Key T. Accuracy of self-reported waist and hip measurements in 4492 EPIC-Oxford participants. Public Health Nutr. 2004;7:723-727.
20. Secretaría de Salud. Norma Oficial Mexicana NOM-006-SSA3-2011 Para la práctica de la anestesiología. México; 2012.

21. Secretaría de Salud. Norma Oficial Mexicana NOM-004-SSA3-2012 del expediente clínico. 2012.

22. IMSS Dirección de Prestaciones Médicas. Procedimiento para la planeación, programación, atención pre-operatoria, trans-operatoria y post-operatoria en las Unidades Médicas Hospitalarias de tercer nivel de atención 2430-003-006. México; 2012.

23. Moore P, Gómez G, Kurtz S, Vargas A. La comunicación médico - paciente: ¿Cuáles son las habilidades efectivas? Rev Med Chile. 2010;138:1047-1054.

24. Taylor C, Dominguez J, Habib A. Obesity and obstetric anesthesia: Current insights. Local Reg Anesth. 2019;12:111-1124.

25. Vaswani PR, Balachandran L. Pregnancy outcomes in a population with high prevalence of obesity: how bad is it? Clin Epidemiol Glob Heal [Internet]. 2013;1:5-11. Available from: https://linkinghub.elsevier.com/ retrieve/pii/S2213398412000103

26. Chu S, Kim S, Schmid C, Dietz P, Callaghan W, Lau J, et al. Maternal obesity and risk of cesarean delivery: A meta-analysis. Obes Rev. 2007;8:385-394.

27. Fisher K, Arnholt A, Douglas M, Vandiver S, Nguyen D. A randomized trial of the traditional sitting position versus the hamstring stretch position for labor epidural needle placement. Anesth Analg. 2009;109:532-534.

28. Afolayan JM, Areo PO, Adegun PT, Ogundipe KO, Filani AB. Comparison of ease of induction of spinal anaesthesia in sitting with legs parallel on the table versus traditional sitting position. Pan Afr Med J. 2017;28:223.

29. de Vignemont F. Body schema and body image--pros and cons. Neuropsychologia. 2010;48:669-680.

30. Morasso P, Casadio M, Mohan V, Rea F, Zenzeri J. Revisiting the bodyschema concept in the context of whole-body postural-focal dynamics. Front Hum Neurosci. 2015;9:83.

31. Cooperstein R, Truong F. Systematic review and meta-analyses of the difference between the spinal level of the palpated and imaged iliac crests. J Can Chiropr Assoc. 2019;63:26-35.

32. Cooperstein R, Truong F. Would adopting a revised landmark rule for the spinal level of the iliac crests improve the accuracy of lumbar level identification? J Can Chiropr Assoc. 2017;61:106-120.

33. Sahin T, Balaban O, Sahin L, Solak M, Toker K. A randomized controlled trial of preinsertion ultrasound guidance for spinal anesthesia in pregnancy: outcomes among obese and lean parturients: ultrasound for spinal anesthesia in pregnancy. J Anesth. 2014;28:413-419.

34. Sahin T, Balaban O. Lumbar ultrasonography for obstetric neuraxial blocks: sonoanatomy and literature review. Turk J Anaesthesiol Reanim. 2018;46:257-267.

35. Secretaría de Salud, Instituto Nacional de Salud Pública, Instituto Nacional de Estadística y Geografía. Encuesta Nacional de Salud y Nutrición ENSANUT 2018. Presentación de resultados [Internet]. 2019 [citado 7 de agosto de 2020]. Disponible en: https://ensanut.insp.mx/encuestas/ ensanut2018/doctos/informes/ensanut_2018_presentacion_resultados.pdf

36. Choi J, Germond L, Santos A. Obstetric Regional Anesthesia [Internet]. NYSORA-The New York School of Regional Anesthesia. [cited 2020 Aug 18]. Available from: https://www.nysora.com/foundations-of-regionalanesthesia/sub-specialties/obstetric/obstetric-regional-anesthesia/

37. Faure E, Moreno R, Thisted R. Incidence of postdural puncture headache in morbidly obese parturients. Reg Anesth. 1994;19:361-363.

38. Loubert C, Fernando R. Cesarean delivery in the obese parturient: anesthetic considerations. Women's Heal. 2011;7:163-179.

39. Arnolds D. COVID-19 and Obstetric Anesthesia [Internet]. ASRA American Society of Regional Anesthesia and Pain Medicine. 2020. Available from: https://www.asra.com/guidelines-articles/originalarticles/article-item/asra-news/2020/07/02/covid-19-and-obstetricanesthesia 